fund exchanges without a research content, and the differences that still exist in the systems for specialist training in France and Britain may cause problems in deciding which posts in the two countries are comparable. The Ministère des Affaires Étrangères in France has recently been prepared to fund visits from fairly senior clinicians: at present there are two British doctors in Paris and one in Lyon working with full responsibility and paid at senior registrar level. Opportunities of this kind are not yet being fully used. For the time being, however, the advice for a British doctor wishing to work in a clinical rather than a research setting at SHO or registrar level in France is to make personal contact with a hospital specialist there who would be willing to sponsor his candidature.

\section{Overview}

In both contexts-clinical work and research-the impression on both sides of the Channel seems to be that interchange is far easier than most doctors believe. Anyone who would like the experience of working in France (or, in the other direction, Britain) should be able to arrange an exchange for any period between a few days and a year. Information about possible research exchanges is available from the MRC, INSERM, and CNRS ; and possible sources of travel funds and grants for longer stays include the British Council, the Royal Society, the Ciba Foundation, and the Wellcome Trust, and in France the Ministère des Affaires Étrangères or the British Council (Paris).

\title{
Guidelines to aid ethical committees considering research involving children
}

\author{
Working Party on Ethics of Research in Children
}

The British Paediatric Association set up in 1978 a Working Party on Ethics of Research in Children (members Professor F Cockburn, Professor J A Dudgeon, Dr D M T Gairdner, Dr A D M Jackson). The following "Guidelines to aid ethical committees considering research involving children"* proposed by the working party have been accepted by the council of the BPA and have been published in the January issue of Archives of Disease in Childhood (1980;55:75-7).

These guidelines presume that four premises are accepted.

That research involving children is important for the benefit of all children and should be supported and encouraged and conducted in an ethical manner.

That research should never be done on children if the same investigation could be done on adults.

That research which involves a child and is of no benefit to that child (non-therapeutic research) is not necessarily either unethical or illegal. ${ }^{1}$

That the degree of benefit resulting from a research should be assessed in relation to the risk, of disturbance, discomfort, or pain-the risk: benefit ratio.

\section{Defining "risk"}

Risk, in this context, means the risk of causing physical disturbance, discomfort or pain, or psychological disturbance to the child or his parents, rather than the risk of serious harm, which no ethical committee would countenance in any case.

Negligible risk-Risk less than that run in everyday life.

Minimal risk-Risk questionably greater than negligible risk.

More than minimal risk.

*A child in this context is taken to include the infant from the time of birth (but not the fetus). Thereafter, an individual remains a minor until 18. The Family Law Reform Act (1969) provides that a minor who has attained the age of 16 has capacity to consent to surgical, medical, or dental treatment (which 'includes any procedure undertaken for the purpose of diagnosis .... and applies to any procedure ... which is ancillary to any treatment as it applies to that treatment'). This statute does not deal with consent for other procedures, e.g. nontherapeutic research, and therefore the general law applies to all minors until 18. Such law does not recognise any 'age of consent', for capacity to consent depends on the child's intellectual capability and the complexity of the procedure; age is simply one factor to be taken into account. $^{2}$

\section{Defining "benefit" \\ Non-therapeutic research}

(a) The procedure is of no benefit to the subject but may benefit the health and welfare of other children or adults. A special case, but an important one, is if the subject suffers from a disorder and the research aims to benefit others suffering from a similar disorder.

(b) The procedure is of no benefit to the subject but may add to basic biological knowledge-for example, normal values; aging.

\section{Therapeutic research}

The procedure is of potential benefit to the subject.

\section{Applying the risk:benefit principle in non-therapeutic research}

Procedures requiring ethical judgments are usually those which are without benefit to the subject-non-therapeutic research. Most such procedures will fall into one of the following three categories.

(1) The procedure is either $(a)$ part of the ordinary care of the infant or child (weighing, measuring, feeding), or (b) involves the non-invasive collection of samples-for example, urine, faeces, saliva, hair, or nail clippings, or, at birth, cord blood or placental tissue.

Risk is here likely to be negligible-for example, test weighing a breast-fed baby as part of a study aimed to promote breast feeding.

(2) The procedure involves invasive collection of samplesfor example, blood, cerebrospinal fluid, or biopsy tissue-taken from a child who is undergoing treatment. The sample used for research may be $(a)$ an additional amount to that required on clinical grounds; or (b) not an ordinary part of the child's treatment-for example, collection of biopsy material during a surgical operation.

Risk in (a) might be either negligible or minimal; (b) might be negligible, minimal, or more than minimal.

Examples-In cystic fibrosis, a research might be considered reasonable which involved an affected child having a sweat test that needed twice as much sweat as required for purely 
diagnostic purposes. The added discomfort to the child might be assessed as negligible. If in addition a venepuncture was required, this might be judged to put the risk of discomfort and pain into the minimal risk category. But the potential benefit to other child sufferers from this common and serious disease might be deemed such as to make the risk:benefit ratio acceptable.

During the course of an operation for hernia, a fragment of skin from the incision might be required for a research involving tissue culture. The risk could be judged negligible, so that even if the research was not expected to have any direct clinical benefit but only to add to basic biological knowledge, it might be acceptable.

During the course of an abdominal operation, a renal biopsy might be taken for research purposes. The risk here would be judged more than minimal and the benefit would have to be very large to justify it. But suppose the research aimed to resolve the problem of rejection of transplanted kidneys, with resulting lifesaving consequences both for children and adults with renal failure; this might be considered a benefit of sufficient magnitude to justify the risk.

(3) The procedure is quite apart from the necessary care or treatment of the child. For example, blood sampling; passage of oesophageal tube for pressure recording; application of face mask for respiration studies; placement of infant in plethysmograph chamber for thermal or respiratory studies; needle biopsy of skin or fat; or $x$-ray or isotope studies (see below).

The risk might be negligible, minimal, or more than minimal. The benefit, as defined above in relation to non-therapeutic research, may fall within either the definition $(a)$ or $(b)$. If it comes under definition (a), the risk should, to be acceptable, probably be either negligible or minimal. If the benefit comes under definition (b), the risk should be negligible.

Examples-In thalassaemia, a common and lethal disease, progress might depend on taking blood specimens from both affected and unaffected children. The benefit could be assessed as great, so justifying the risk of causing more than minimal discomfort or pain to the children.

Many diabetic children will develop blindness or other severe eye complications in adult life. A research aimed at eventually learning how to prevent this might require several glucose tolerance tests to be done on a diabetic child, not for his own benefit but for the benefit of other diabetic children. The risk of discomfort or pain to that child would be assessed as more than minimal, but might nevertheless be justified by the potential benefit.

The physiology of the initiation of breathing by the baby at birth is poorly understood, and is of clinical importance because some babies fail to breathe. A study of normal newborn babies' first breath, using a face mask, may be judged to cause minimal risk with a justifiable risk:benefit ratio.

\section{Applying the risk:benefit principle in therapeutic research}

Therapeutic research offers potential benefit to the subject. It includes not only trials of new drugs or procedures but also trials of therapies which, though perhaps widely applied, are yet of unproved value. The risk:benefit principle may still be applicable, the potential benefit as well as the risk relating to the individual subject.

In general, ethical principles in therapeutic research involving children do not usually differ from those applying to adults, except that the age of the subject will often mean that parental understanding and agreement will be required.

In the common type of experiment where two therapies are compared in a controlled trial, two ethical questions are likely to arise.

(1) Is the research necessary? For instance, conventional treatment of a febrile convulsion in a child includes drastic cooling. A research project might question this form of manage- ment and entail a controlled trial. An ethical committee might consider it probable that data already existed enabling the question to be answered. The committee might therefore require the researcher first to provide evidence that the world literature had been effectively searched.

(2) Is the design of the trial such that a statistically significant result will emerge with the use of a minimal number of subjects and in a minimum period? Since one set of children will receive what may eventually turn out to be an inferior therapy, it is ethically imperative that this question be answered in the affirmative.

Examples-Current research in treating leukaemia in children often means comparing two different drug regimens. Since both sets of children receive therapies currently considered acceptable, ethical considerations are mainly confined to ensuring that the design of the trial is statistically sound.

A controlled trial of hyposensitising injections of allergens in asthmatic children differs from the foregoing example in that some children (the controls) receive injections of inactive material. This might at first sight scem ethically questionable. However, the following consideration may lead to such a trial being judged acceptable. Until the result of the trial is known the children in either the treatment or the control group have a chance of gaining an advantage. The active therapy may prove superior and those in the treatment group gain an advantage. If, however, there are unpleasant or harmful side effects from the active therapy, the control group will have gained some advantage by not being exposed to those side effects.

\section{$X$ rays and isotopes}

An authoritative pronouncement on the ethical propriety of irradiating children-that is, the use of $x$ rays or isotopesfor research purposes has recently been given by the International Commission on Radiological Protection. ${ }^{3}$ It states that "the irradiation, for the purposes of such studies (that is, of no direct benefit to the subject) of children and other persons regarded as being incapable of giving their true consent should only be undertaken if the expected radiation is low (for example, of the order of one-10th of the dose-equivalent limits applicable to individual members of the public) and if valid approval has been given by those legally responsible for such persons."

This means, in common parlance, that exposure to $x$ rays could be justifiable where the dosage was comparable to the normal variation in natural irradiation received by, say, individuals living in two different parts of the British Isles. In fact, using modern equipment, a single radiograph might fall well within such dosage limits, and thus be classifiable as a negligible risk.

\section{Parental permission and co-operation. Agreement by the child}

Parental (or guardian's) permission should normally be obtained-with rare exceptions such as the comparison of two treatments for some emergency condition-after explaining as fully as possible the nature of the procedure. Whether or not this should be a signed, witnessed declaration remains debatable. It is an advantage if the parents can be present during the procedure. Although the law in Britain does not recognise an age of consent, children much younger than 16 often have enough understanding to collaborate altruistically in a project.

\section{New drugs: new immunisation procedures}

In general these should be first tested on animals, then on adult volunteers, then on older children able to take part voluntarily in the research, and only then on younger children. 
However, there are instances where this sequence might be inappropriate; for instance in the development of a vaccine against respiratory syncytial virus, where few uninfected subjects may be available above the age of infancy.

\section{British Paediatric Association: Standing Ethics Advisory Committee}

(1) The British Paediatric Association has set up a Standing Ethics Advisory Committee. The function of this Committee will be to offer advice on the ethics of research projects involving children.

(2) The Committee will respond to requests for advice from individuals planning research projects, from local ethical Com- mittees, or from editors of journals, but its opinions will not be binding. Approval of research projects must remain the responsibility of statutory local ethical committees.

(3) The Committee will base its opinions on guidelines drawn up by the British Paediatric Association, and if it is consulted often enough it will in time establish some uniformity of policy for research in children throughout the country.

\section{References}

${ }^{1}$ Dworkin G. Legality of consent to nontherapeutic medical research on infants and young children. Arch Dis Child 1978;53:443-6.

2 Skegg PDG. English law relating to experimentation on children. Lancet 1977;ii:754-5.

${ }^{3}$ International Commission on Radiological Protection. Ann Int Commis Radiol Protect 1977;1:No 3, 37.

\title{
Letter from . . . Western Australia
}

\section{Flying to the rescue}

\author{
BOB MILSTED
}

When Paddy Hannan discovered gold east of Perth in 1893 he started the gold rush which gave birth to the town of Kalgoorlie. It's a mining town, first and last, and has relied for its existence ever since on the world demand for gold or, more recently, nickel, and on a slender pipeline which brings water pumped from Perth 350 miles away. Kalgoorlie boasts the near perfect proportions of 20000 people to one set of traffic lights, and sits like a small island in a huge sea of arid bush. Having arrived in Perth jobless and penniless after four months' driving overland through Asia, the opportunity to work for the Eastern Goldfields section of the Royal Flying Doctor Service was definitely not to be missed. The service employs two doctors, three nurses, and four pilots and covers an area around Kalgoorlie slightly larger than the United Kingdom - surely the largest general practice in the world. The hub is the radio base, which serves as the sole link between the outside world and the isolated sheep stations and Aborigine camps of the Central Desert. The base deals with telegrams and provides a school of the air for children on stations, as well as dealing with medical calls. Each morning at 11 am there is a regular schedule for non-urgent medical inquiries. Each station has a medical chest with a variety of numbered drugs, which they take according to advice received.

"I suggest you take two 93s and a 15," sounds a little strange coming over the air, but the system works very well. Emergency calls after hours trigger a radio telephone in the home of the doctor on call. Diagnosis over the radio takes a little practice, but the decision whether to send out a plane is helped by the knowledge that an emergency call from a station is unlikely to be trivial-perhaps a child with an arm lacerated by farm machinery, or an adult bitten by a snake. The pastoralists, who live up to a 100 miles from their nearest neighbours, survive the rigours of running a station and raising a family in

Kalgoorlie, Western Australia

BOB MILSTED, MD, MRCP, medical officer an unforgiving environment with unfailing good humour. In the process, they seem to become as tough as their lifestyle. The most bizarre call was from a dogger (who baits traps for dingos), who called in to ask whether there was an antidote to strychnine as he had just inhaled some. He survived, despite having a supposedly lethal level of strychnine in his blood.

\section{Snags and kangaroos}

Many people are under the illusion that flying doctors actually pilot the planes. Fortunately, this is no longer the case. Any such romantic notions disappear rapidly when landing on a bush strip at night by the light of car headlights and a few fruit cans filled with kerosene. On one night-landing we touched down only to find ourselves bearing down on a kangaroo. Unperturbed, the pilot lifted the plane over the bemused animal's head and touched down again behind him. The planes are twin-engined Navajos, capable of taking two stretchers, but definitely not the ideal place to carry out emergency procedures. Transporting patients in unpressurised aircraft creates unique problems. An undrained pneumothorax will expand as the atmospheric pressure decreases, as will distended bowel. Patients with respiratory problems may deteriorate, and labouring women seem to progress rather more rapidly than is good for the doctor's nerves. Simple things become difficult; drips stop running and the cuff of an endotracheal tube expands at altitude. The rule is to do whatever is necessary for the patient's safety before taking off. Whenever possible, pregnant mothers are brought to Kalgoorlie two weeks before term to await delivery in the hospital there and avoid mid-air drama. One priority after delivery is, of course, to radio the good news to the husband. The "Bloody beaut!" response of one proud father was an uninhibited expression of delight which, transmitted as it was over the whole network, brightened the day of everyone within a radius of several hundred miles.

The twice-weekly visits to the small towns of Leonora and 